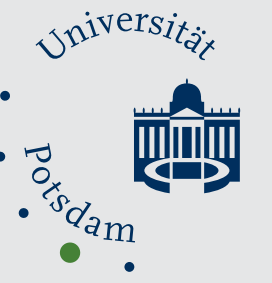

Caroline Fischer

\title{
Neue Talente braucht das Amt
}

Traineeprogramme im öffentlichen Sektor

Suggested citation referring to the original publication:

Talent Management (2018), pp. 63-85

DOI http://dx.doi.org/10.1007/978-3-658-19100-9_4

Postprint archived at the Institutional Repository of the Potsdam University in:

Postprints der Universität Potsdam

Wirtschafts- und Sozialwissenschaftliche Reihe ; 106

ISSN 1867-5808

http://nbn-resolving.de/urn:nbn:de:kobv:517-opus4-430598

DOI https://doi.org/10.25932/publishup-43059 



\title{
Neue Talente braucht das Amt
}

\section{Traineeprogramme im öffentlichen Sektor}

\author{
Caroline Fischer
}

\subsection{Einleitung: Wozu Trainees im öffentlichen Dienst?}

„Good government needs good people“ (Lavigna und Hays 2004, S. 237) - der Staat braucht gutes Personal. Doch Stellenabbau und demografischer Wandel führen zu Rekrutierungsschwierigkeiten und einem Mangel an talentierten Nachwuchskräften in der öffentlichen Verwaltung. Gleichzeitig stellen sich neue Anforderungen an öffentliche Behörden, die eine andere Qualifikation der Beschäftigten nach sich ziehen (Thom und Ritz 2008).

Diese Gemengelage gilt für viele öffentlichen Dienste weltweit. Besonders betroffen sind aber sog. geschlossene Personalsysteme, in denen Barrieren zwischen öffentlichem und privatem Arbeitsmarkt existieren und die häufig mit dem Laufbahnprinzip einhergehen. Das Personal wird hier nicht für eine bestimmte Position rekrutiert und ausgebildet, sondern durchläuft stufenweise eine Laufbahn ${ }^{1}$. In vielen Ländern werden diese starren Beschäftigungsregime jedoch nach und nach flexibilisiert (Demmke 2011; Laegreid und Wise 2007), um auf die genannten Herausforderungen zu reagieren.

Eine Möglichkeit zur Flexibilisierung der Rekrutierung und Ausbildung von Nachwuchskräften stellen Traineeprogramme dar, die konzeptionell als Maßnahmen von Talent Management eingeordnet werden können. Diese Programme, die im Privatsektor

${ }^{1}$ Für die Unterscheidung in offene und geschlossene Personalsysteme und Laufbahn- und Positionssysteme siehe beispielsweise Reichard und Schröter (2009, S. 20-22).

C. Fischer ( $\square)$

Professur Public und Nonprofit Management der Universität Potsdam, Potsdam, Deutschland

E-Mail: cpath@uni-potsdam.de 
schon seit einigen Jahrzehnten etabliert sind, richten sich i. d. R. an Hochschulabsolventen, die eine Grundlagenausbildung für die spätere Übernahme von Führungsaufgaben erhalten (Thom 1987). In Deutschland werden Traineeprogramme im öffentlichen Sektor seit einigen Jahren v. a. auf kommunaler und Landesebene erprobt. Sie ermöglichen Nichtjuristen den Einstieg in die höhere Beamtenlaufbahn. Das unterstützt die fachliche Diversifizierung des deutschen öffentlichen Personals, für das nach wie vor ein „Monopol von Juristen“ beklagt wird (Reichard 2013, S. 291). Eine solche fachliche Diversität kann sich positiv auf die Leistung der Organisationen auswirken (Horwitz und Horwitz 2007), insbesondere, wenn es um innovatives Verwaltungshandeln geht (Bell et al. 2011).

Im Folgenden sollen zunächst die Besonderheiten eines öffentlichen Talent Managements dargelegt und aufgezeigt werden welche Formen der Rekrutierung und Ausbildung von talentierten Nachwuchskräften im deutschen öffentlichen Dienst (bisher) vorherrschen. Anschließend wird ein Überblick über Traineeprogramme der deutschen öffentlichen Verwaltung gegeben. Hierbei werden Ergebnisse einer quantitativen Befragung von Trainees und deren Führungskräften im öffentlichen Dienst vorgestellt und die Unterschiede zu privaten Traineeprogrammen herausgearbeitet. ${ }^{2}$

\section{2 Öffentliches Talent Management}

Talent Management ist nach wie vor ein junges Forschungsfeld, in dem keine abgeschlossene Theoriebildung existiert und Auseinandersetzungen über Definitionen herrschen (Lewis und Heckman 2006; Collings und Mellahi 2009; Thunnissen et al. 2013a). „Nearly every article written on the topic begins with handwringing over the conceptual boundaries of the term" (Cappelli und Keller 2014, S. 306). Diese wurden in zahlreichen Reviews aufgearbeitet (Lewis und Heckman 2006; Vaiman und Collings 2013; Dries 2013; Gallardo-Gallardo et al. 2015). Die meisten Konfliktlinien in der Diskussion um Talent Management entspinnen sich an der Definition von Talent. Ist es angeboren oder erworben und damit erlernbar; sind Talente rar oder trägt jeder Talent in sich; ist Talent transferierbar oder kontextgebunden (Dries 2013)? Eben diese Konfliktlinien werden auch in der Diskussion um ein passfähiges öffentliches Talent Management relevant.

Die empirische Lage zum Talent Management im öffentlichen Sektor ist dürftig. Die hier existierenden Ergebnisse sind eher beschreibender Natur und stammen zum größten Teil aus dem angloamerikanischem Raum (Harris und Foster 2010; Glenn 2012; Macfarlane et al. 2012; Berman et al. 2013; Powell et al. 2013; Rutledge et al. 2016; Smith 2015) oder beschäftigen sich mit Entwicklungs- und Schwellenländern (Poocharoen und Lee 2013; Gholipour et al. 2015; Suk Kim und Kotchegura 2016). Für den deutschen Kontext liegen bisher keine Studien zu öffentlichem Talent Management vor. Die Ergebnisse der Studien mit Länder- und Organisationsfokus zeigen aber, dass diese Kontexte

\footnotetext{
${ }^{2}$ Für die Mitarbeit an diesem Beitrag danke ich ganz herzlich Anastasios Mavrogiannis.
} 
Einfluss auf die Ausgestaltung und Wirkung von Talent Management haben und daher untersucht werden müssen.

\subsubsection{Elitärer Talentbegriff}

Wenn Talent als angeboren betrachtet wird, zieht das in Maßnahmen des Talent Managements einen Fokus auf die Auswahl von Talenten nach sich. Dies kann durch externe Rekrutierung oder interne Identifizierung geschehen. Wenn Talent als erlernbar betrachtet wird, dann liegt der Fokus eher auf Personalentwicklungsmaßnahmen. Meyers et al. (2013) schlagen in der Frage der Erlernbarkeit von Talent ein Kontinuum vor, in dem eine Kombination beider Annahmen möglich ist: es muss Potenzial angeboren sein, das zu einem Talent entwickelt werden kann.

Wird Talent oder Potenzial als angeboren betrachtet, entspricht dies auch der Position, dass Talent rar ist und nicht jede Person ein solches in sich trägt. Es wird dann von einem exklusiven Talent Management gesprochen, denn nicht jeder Beschäftigte kann Teil der Maßnahmen sein, wie es in einem inklusiven Talent Management der Fall wäre (Dries 2013). Im exklusiven Talent Management ist häufig von der Entwicklung eines Talentpools die Rede, aus dem Nachwuchskräfte für strategisch wichtige Positionen rekrutiert werden.

Talent Management ist dann ,less egalitarian, and more elitist by definition“ (Dries 2013, S. 274). Der Talentmanager ist im Gegensatz zum Personalreferenten eben kein Vollsortimenter (Jäger 2009). Gerade im öffentlichen Sektor scheint das aber problematisch: ,people often fear that talent management will cut across equality of opportunity and the transparent processes“ (Garrow und Hirsh 2008, S. 390). Eine exklusive Talentförderung kann von Kollegen als unfair wahrgenommen werden und entsprechende Reaktionen hervorrufen (Ritz und Thom 2011; Sheehan und Anderson 2015). Björkman et al. (2013) zeigen z. B., dass Mitarbeiter, die nicht als Talente entdeckt wurden, sich weniger mit ihrem Team identifizieren und eine höhere Kündigungsbereitschaft aufweisen. Dabei scheint eine faire Verfahrensweise bei der Identifikation der Talente entscheidend. So zeigten Gelens et al. (2014) auf, dass beispielsweise als gerecht empfundene Beförderungen die Arbeitszufriedenheit (positiv) beeinflussen, nicht aber die (Nicht-)Identifikation als Talent an sich. Zudem wird deutlich, dass Mitarbeiter, die die Verfahren im Zusammenhang mit der Identifikation von Talenten als gerecht empfinden, sich in ihrer Arbeit mehr bemühen, als diejenigen, die die Verfahren als unfair empfinden, unabhängig davon, ob diese Mitarbeiter selbst als Talent identifiziert wurden (Gelens et al. 2014). Einige Forscher appellieren daher verstärkt für eine bessere Balance zwischen organisationalen und individuellen Zielen von Talent Management (Collings et al. 2015), sodass dieses passfähiger zur Organisation, deren Kultur und Mitarbeitern wird. 


\subsubsection{Transfer von Talent}

Die Transferierbarkeit von Talent wird dann relevant, wenn Talent als angeboren betrachtet wird und ein Fokus auf der externen Rekrutierung liegt (Dries 2013). Es ist die Frage nach „grow“ oder „buy“ von Talenten (Ashton und Morton 2005). Das interne Entwickeln von Talenten (,grow“) ist dabei zumeist kostengünstiger, aber langwieriger; die externe Rekrutierung (,buy“) schneller, aber mitunter weniger passfähig (Cappelli 2008).

Das Einkaufen und damit der Transfer von Talent wird problematisiert, weil zur Debatte steht, ob ein Mitarbeiter aufgrund seines Talents gute Leistungen erbringt, oder ob diese (auch) aus entsprechenden Bedingungen am Arbeitsplatz wie einem harmonisierenden Team oder entsprechenden Prozessen resultieren. Es ist also die Frage, ob sich Talent aus „general human capital“ oder eben auch „firm-specific human capital“ begründet. Groysberg et al. (2008) haben so gezeigt, dass Talente noch fünf Jahre nach einem Wechsel in eine andere Organisation schlechtere Leistungen erbringen, als Kollegen, die die Organisation nicht gewechselt haben.

Wenn die Mitnahme von Talent schon bei einem Organisationswechsel im gleichen Sektor problematisch ist, dürfte sie bei einem Wechsel vom privaten in den öffentlichen Sektor noch schwieriger sein. Es wird daher häufig infrage gestellt, ob ein in der Privatwirtschaft erfolgreicher Beschäftigter im öffentlichen Dienst auch ein Talent sein kann. Dies zielt zum einen auf fehlendes sektorspezifisches Wissen ab, aber auch auf die Sozialisation im öffentlichen Bereich, also das Kennen und Teilen vorherrschender Werte, die sich durchaus vom Privatsektor unterscheiden können.

In Staaten mit eher geschlossenen öffentlichen Personalsystemen herrschen daher Laufbahnkarrieren vor. In diesen werden, häufig auch aufgrund fehlender anderer Belohnungsmöglichkeiten, Positionen intern durch Beförderung besetzt. ${ }^{3}$ Abgesehen von den Staatssekretären werden etwa in Deutschland $75 \%$ der leitenden Führungskräfte in den Ministerien intern aus dem öffentlichen Dienst rekrutiert (Bogumil und Jann 2009). Übergänge vom privaten in den öffentlichen Sektor gestalten sich daher schwierig und insbesondere in Führungspositionen ist der Zugang für Quereinsteiger selten (Kuhlmann und Wollmann 2013).

\subsubsection{Bisherige Formen der Rekrutierung und Ausbildung von Nachwuchs im öffentlichen Dienst}

Im deutschen öffentlichen Dienst im engeren Sinn, also beispielsweise nicht öffentlich beschäftigte Lehrer oder Krankenhausmitarbeiter, werden nach wie vor nach klassischen Mustern Nachwuchskräfte rekrutiert und ausgebildet. Grundsätzlich liegen unterschiedliche

\footnotetext{
${ }^{3}$ Bidwell und Keller (2014, S. 105) haben das auch für private Unternehmen mit Laufbahnstrukturen gezeigt.
} 


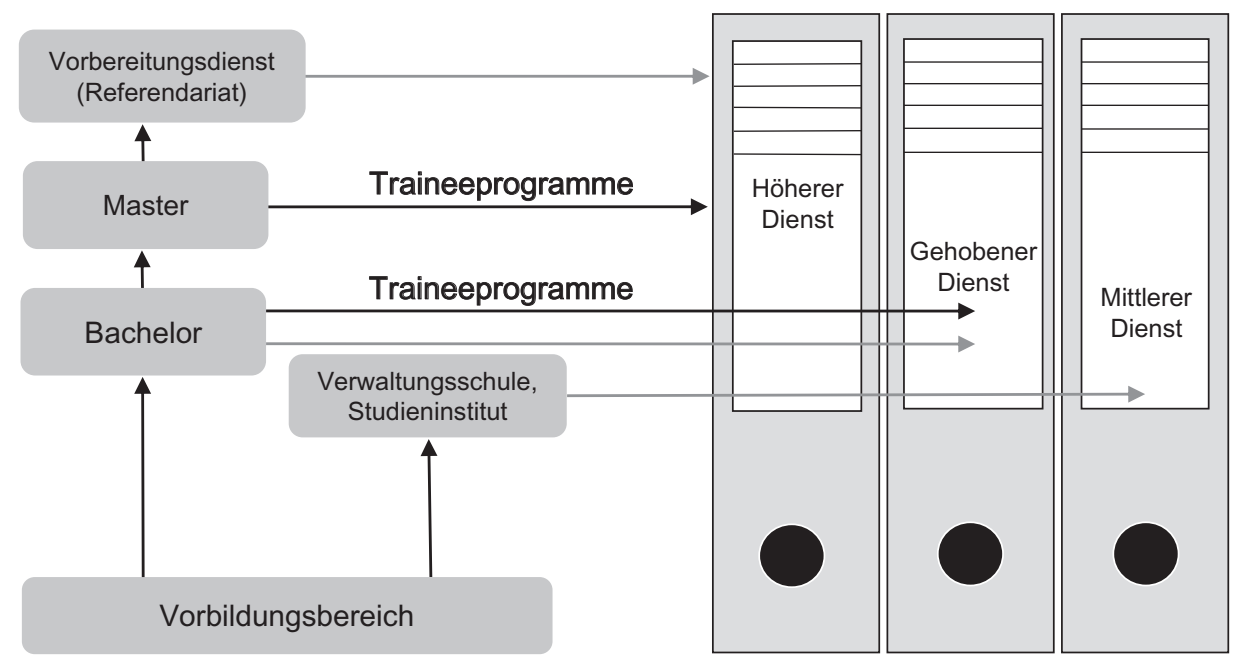

Abb. 4.1 Zugangsweise in den deutschen öffentlichen Dienst. (In Anlehnung an Reichard und Röber 2012, S. 14)

Ausbildungsvarianten für Tarifbeschäftigte im öffentlichen Dienst und Beamte vor ${ }^{4}$ und die Hoheit über die Verwaltungsausbildung liegt dezentral bei den entsprechenden Verwaltungsebenen (Kuhlmann und Wollmann 2013). Die Abb. 4.1 zeigt, welche Ausbildungswege für welche Laufbahn in der öffentlichen Verwaltung qualifizieren. ${ }^{5}$ Für den mittleren Dienst werden in einer Berufsausbildung Verwaltungsfachangestellte ausgebildet, die als Tarifbeschäftigte Sachbearbeiterpositionen besetzen können. Die entsprechende Beamtenausbildung ähnelt dieser Berufsausbildung inhaltlich, es findet jedoch kein Berufsschulunterricht statt (Reichard und Röber 2012).

Für den gehobenen Dienst qualifizieren verschiedene verwaltungsbezogene Bachelorstudiengänge an Fachhochschulen. Die Ausbildung erfolgt dabei an internen Fachhochschulen der Länder oder externen allgemeinen Fachhochschulen (Reichard und Röber 2012).

Für den höheren Dienst qualifiziert grundsätzlich ein zweiter Studienabschluss einer Universität. Hochschulabsolventen durchlaufen für die Einstellung in den öffentlichen Dienst dann i. d. R. eine Vorbereitungsphase. Für Juristen, die nach dem an der Universität abgelegten ersten Staatsexamen ein Rechtsreferendariat absolvieren, ist dies die sog. Verwaltungsstation innerhalb des Referendariats. Dieser Form des Referendariats ist das

\footnotetext{
${ }^{4}$ Für diese Dualität im deutschen öffentlichen Dienst siehe beispielsweise Reichard und Schröter (2009, S. 27). Beamten ist dabei die Ausübung hoheitlicher Aufgaben vorbehalten und sie stehen in einem öffentlich-rechtlichen Dienstverhältnis auf Lebenszeit. Tarifbeschäftigte hingegen sind privatrechtlich angestellt und grundsätzlich kündbar (Kuhlmann und Wollmann 2013).

${ }^{5}$ Die Benennung der Laufbahnen in mittlerer, höherer und gehobener Dienst wird nicht mehr in allen Laufbahnordnungen verwendet. So gehören im Land Berlin seit der Reform des Laufbahngesetzes in 2011 alle Laufbahnen die einen Hochschulabschluss erfordern zur zweiten Laufbahngruppe, alle übrigen zur ersten Laufbahngruppe. Im Folgenden werden der Verständlichkeit halber dennoch die althergebrachten Laufbahnbezeichnungen verwendet.
} 
Wirtschafts- oder Regierungsreferendariat nachempfunden, das sich an wirtschafts- und sozialwissenschaftliche Bewerber richtet. Mit einem solchen Referendariat können diese eine Befähigung für die höhere Beamtenlaufbahn erlangen. Hier werden i. d. R. innerhalb von zwei Jahren verschiedene Verwaltungsbereiche durchlaufen und eine begleitende Fortbildung absolviert.

Traineeprogramme kommen in Deutschland nach und nach für den gehobenen und den höheren Dienst zum Einsatz. Sie bieten formell die Möglichkeit, auch Nachwuchskräfte, die sonst nur als Tarifbeschäftigte angestellt werden können, zu verbeamten. Sie ersetzen damit die Funktion der Wirtschafts- oder Regierungsreferendariate, sind z. T. aber auch zur weiteren Qualifizierung von Juristen vorgesehen, die schon eine Laufbahnbefähigung erworben haben. Die Programme werden zudem nicht immer zum Erreichen der Laufbahnbefähigung eingesetzt, sondern insbesondere in der Kommunalverwaltung, wo insgesamt weniger Beamtenstellen zur Verfügung stehen und Tarifbeschäftigte dominieren, auch zur Vorbereitung von Nachwuchskräften, die tarifbeschäftigt übernommen werden.

Traineeprogramme und Referendariate sind sich im Aufbau ähnlich. Beide Ausbildungsformate beinhalten Praxisstationen in verschiedenen Verwaltungsbereichen und begleitende Fortbildungsangebote. Ebenso dienen beide zur Erlangung der Laufbahnbefähigung. Im Unterschied zum Referendariat schließen Traineeprogramme aber per definitionem nicht mit einer Laufbahnprüfung ab. Am deutlichsten unterscheiden sich beide Formate in der Stellung der Teilnehmer. Trainees haben meist einen privatrechtlichen Status als Tarifbeschäftigte, im Referendariat sind die Teilnehmer hingegen als Beamtenanwärter öffentlich-rechtlich beschäftigt. Das wirkt sich auch auf die Bezahlung aus: Trainees werden dabei i. d. R. höher bezahlt als Beamtenanwärter. ${ }^{6}$

\subsection{Traineeprogramme im öffentlichen Sektor}

„Die Zielsetzung eines systematischen Talent Managements besteht darin [...] die ,Talent-Pipeline' proaktiv und kontinuierlich zu füllen, damit auch in Zukunft die richtigen Mitarbeiter mit den richtigen Kompetenzen zur richtigen Zeit am richtigen Ort eingesetzt werden können“ (Heller und Ruf 2009, S. 121). Genau dieses Ziel können Traineeprogramme erfüllen. Mit ihnen kann ein (Teil eines) Talentpools aufgebaut bzw. die Talentpipeline gefüllt werden. In Abb. 4.2 wird deutlich, wie Traineeprogramme als Teil von Talent Management einer Organisation eingeordnet werden können.

Traineeprogramme dienen der organisationsspezifischen Nachwuchsförderung. Sie werden im Personalmanagement als Maßnahmen der „Personalentwicklung into-the-job“ eingeordnet (Scholz 2000). Sie vereinen Komponenten einer Personalentwicklung on-the-job, wie etwa Projektarbeit, und off-the-job, wie etwa Fortbildungen, und zeichnen sich durch

\footnotetext{
${ }^{6}$ Beispielsweise waren die Bezüge für Beamtenanwärter beim Land Berlin, Eingangsamt A13 im Jahr 2015 1166,30 EUR (brutto, Grundbetrag; dbb Berlin 2015a). Der Lohn von Trainees im Land Berlin, E13 erstes Einstiegsamt lag im Jahr 2015 hingegen bei 3386,71 EUR (brutto; dbb Berlin 2015b).
} 


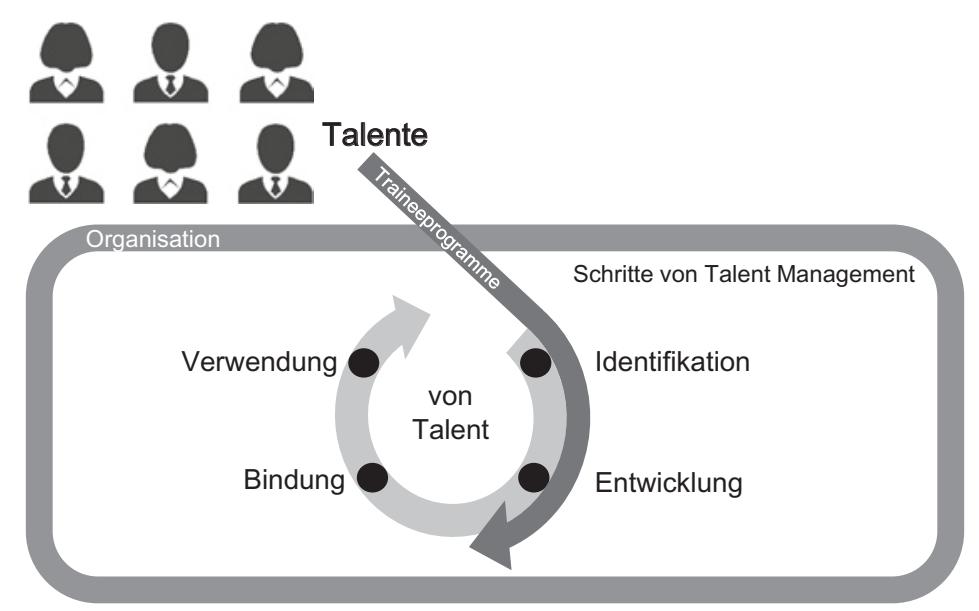

Abb. 4.2 Traineeprogramme als Teil von Talent Management

Jobrotation aus (Gulden 1996). Die Programme unterscheiden sich durch ihr planvolles Vorgehen, ihre didaktische Struktur und die Anwendung von Einzelmaßnahmen der Personalentwicklung (Thom und Friedli 2008). Sie eignen sich daher erst ab einer Organisationsgröße, in der ausreichend Nachwuchskräfte als Programmteilnehmer zur Verfügung stehen.

Indem diese Programme sich nur auf einen engen Teilnehmerkreis konzentrieren, gehören sie zum exklusiven Talent Management. Die Trainees werden von außen rekrutiert, aber dann intern aufgebaut, sodass hier von einem Hybrid des Grow- und des Buy-Ansatzes gesprochen werden kann. Dieser Kompromiss scheint insbesondere für den öffentlichen Dienst sinnvoll, um Akzeptanzproblemen aufgrund der Exklusivität der Talentförderung vorzubeugen und Transferprobleme zu vermeiden.

\subsubsection{Arten und Ziele von Traineeprogrammen}

Es können vier Grundmodelle von Traineeprogrammen unterschieden werden, die in der Praxis jedoch nicht immer so idealtypisch vorliegen. Im klassischen ressortübergreifenden Traineeprogramm durchlaufen die Teilnehmer verschiedene Bereiche in einer Organisation und werden so zu Generalisten ausgebildet (Gulden 1996). Im ressortübergreifenden Traineeprogramm mit Fachausbildungsphase verbringt der Trainee ,im Anschluss an das verkürzte Rotationsprogramm eine deutlich längere Zeit in einem der vorher durchlaufenen Ressorts und vertieft hier seine Kenntnisse“ (Thom und Friedli 2008, S. 16). Ressortbegrenzte Traineeprogramme hingegen finden lediglich in einem Bereich einer Organisation statt, in dem die Trainees verschiedene Fachbereiche oder Abteilungen durchlaufen (Thom und Friedli 2008). Ziel dieser Programme ist es, die Teilnehmer zu Ressortspezialisten auszubilden (Nesemann 2012, S. 40). Wenn die Teilnehmer hingegen 
vornehmlich in Projekten arbeiten, so wird von projektbezogenen Traineeprogrammen gesprochen (Thom und Friedli 2008).

Alle diese Varianten von Traineeprogrammen haben ein gemeinsames Ziel: die Versorgung einer Organisation mit qualifizierten Nachwuchskräften, das in der Forschung meist in fünf Teilziele untergliedert wird: Ausbildungserfolg, Informationsergiebigkeit, Sozialisationserfolg, Imageverbesserung und Nachhaltigkeit (Thom und Friedli 2008; Nesemann 2012).

Der Ausbildungserfolg bemisst sich am Zuwachs von fachspezifischem und methodischem Wissen und sozialer Kompetenz während der Ausbildung im Programm (Nesemann 2012). Der Fokus liegt dabei auf dem fachlichen Ausbildungserfolg, für den sowohl der Zugewinn an spezifischem Wissen eines Ressorts als auch der Zugewinn an bereichsübergreifendem Wissen entscheidend ist (Cordes 2000).

Informationsergiebigkeit hingegen meint den Zuwachs an Wissen aus Sicht der Organisation und zwar über die Programmteilnehmer als Kandidaten für Führungspositionen. So soll das Auswahlrisiko für die spätere Stellenbesetzung reduziert werden (Thom 1987).

Für eine erfolgreiche Sozialisation der Trainees in der Organisation sollen diese organisationale Werte und Verhaltenserwartungen kennen und anwenden und mit Kollegen vernetzt sein. Eine positive Sozialisation wirkt sich dann ,,auf Performance, Zufriedenheit sowie organisationales Commitment der Nachwuchskräfte“ (Nesemann 2012, S. 51) aus. Eine fehlende Sozialisation kann hingegen zum Verlassen der Organisation führen und wirkt damit negativ auf die Nachhaltigkeit eines Traineeprogramms (Nerdinger 2014).

Schließlich verfolgen Organisationen mit Traineeprogrammen eine Imageverbesserung. Extern sollen die Programme das Image des Anbieters als Arbeitgeber verbessern und der Organisation Vorteile in der Rekrutierung von talentierten Nachwuchskräften sichern (Nesemann 2012). Das interne Image eines Traineeprogramms bei den anderen Mitarbeitern ist hierfür eine wichtige Basis. Ein gutes internes Image deutet auf die Passfähigkeit des Programms zur Organisation und deren Werte hin, die gerade im Fall exklusiver Maßnahmen von Talent Management entscheidend ist.

Aufgrund der Kosten eines Traineeprogramms liegt es im Interesse der Anbieter, dass die Trainees den Organisationen nach Abschluss des Programms erhalten bleiben. Hieraus kann die Nachhaltigkeit von Traineeprogrammen bestimmt werden.

\subsubsection{Traineeprogramme im deutschen öffentlichen Dienst}

In Deutschland existieren in den verschiedenen Verwaltungsebenen zwar Traineeprogramme, bezeichnen allerdings z. T. sehr unterschiedliche Arten der Personalentwicklung. ${ }^{7}$ So kann beispielsweise die als Traineeprogramm benannte Führungskräftefortbildung des Landes Thüringen für zukünftige Referatsleiter nicht als Traineeprogramm im eigentlichen

\footnotetext{
${ }^{7}$ Diese Problematik wird auch im Privatsektor bemängelt, wo unter dem Label Traineeprogramm mitunter lediglich Direkteinsteiger schlechter bezahlt werden (Rippler et al. 2013).
} 
Sinn bezeichnet werden. Das Programm richtet sich nicht an Nachwuchskräfte, sondern Berufserfahrene und dient lediglich der internen Personalentwicklung. Das gleiche gilt für das Traineeprogramm der niedersächsischen Oberfinanzdirektion, das Juristen innerhalb eines Jahres zu Führungsaufgaben fortbildet oder das Programm der Bundesagentur für Arbeit. Aufgrund dieser begrifflichen Unschärfe existieren sehr unterschiedliche Angaben zur Verbreitung dieser Programme in der deutschen Verwaltung (Görtler und Gourmelon 2015; Reichard und Röber 2012; Lück 2011). In Tab. 4.1 findet sich eine Übersicht über Traineeprogramme im deutschen öffentlichen Dienst.

Tab. 4.1 Ausgewählte Traineeprogramme im deutschen öffentlichen Dienst. (In Anlehnung an Fischer 2016, S. 256)

\begin{tabular}{|c|c|c|c|c|}
\hline Ort & Form & $\begin{array}{l}\text { Dauer } \\
\text { (Monate) }\end{array}$ & Teilnehmer & Besonderheiten \\
\hline \multicolumn{5}{|l|}{ Bund } \\
\hline Bundesbank & $\begin{array}{l}\text { Rotation mit } \\
\text { Fachausbildung }\end{array}$ & 12 & $\begin{array}{l}\text { Wirtschaftswissen- } \\
\text { schaftler }\end{array}$ & $\begin{array}{l}\text { Einsatz bundesweit, in Haupt- } \\
\text { verwaltungen und Filialen }\end{array}$ \\
\hline \multicolumn{5}{|c|}{ Bundesländer } \\
\hline Berlin & $\begin{array}{l}\text { Rotation mit } \\
\text { Fachausbildung }\end{array}$ & 24 & $\begin{array}{l}\text { Wirtschafts- und } \\
\text { Sozialwissenschaftler }\end{array}$ & $\begin{array}{l}\text { Neben dem Programm für } \\
\text { den höheren Dienst seit } 2016 \\
\text { auch Programm für den } \\
\text { gehobenen Dienst (Bache- } \\
\text { lorabsolventen) }\end{array}$ \\
\hline Bremen & $\begin{array}{l}\text { Klassisches } \\
\text { Rotationspro- } \\
\text { gramm }\end{array}$ & Bis 36 & $\begin{array}{l}\text { Wirtschaftswissen- } \\
\text { schaftler }\end{array}$ & $\begin{array}{l}\text { Sog. Nachwuchspool, flexibel } \\
\text { in Ablauf und Inhalt }\end{array}$ \\
\hline Hamburg & $\begin{array}{l}\text { Klassisches } \\
\text { Rotationspro- } \\
\text { gramm }\end{array}$ & 18 & $\begin{array}{l}\text { Wirtschafts- und } \\
\text { Sozialwissenschaftler }\end{array}$ & $\begin{array}{l}\text { Station in einem Unterneh- } \\
\text { men oder der EU-Vertretung } \\
\text { (Hanse Office, Brüssel) } \\
\text { möglich }\end{array}$ \\
\hline Hessen & $\begin{array}{l}\text { Klassisches } \\
\text { Rotationspro- } \\
\text { gramm }\end{array}$ & 30 & Juristen & $\begin{array}{l}\text { Station in einem Unterneh- } \\
\text { men der Privatwirtschaft }\end{array}$ \\
\hline \multicolumn{5}{|c|}{ Kommunen } \\
\hline Ingolstadt & $\begin{array}{l}\text { Klassisches } \\
\text { Rotationspro- } \\
\text { gramm }\end{array}$ & 24 & $\begin{array}{l}\text { Juristen, Wirtschafts- } \\
\text { und Sozialwissen- } \\
\text { schaftler, Ingenieure }\end{array}$ & $\begin{array}{l}\text { Unterschiedliche Programm- } \\
\text { varianten für Interne und } \\
\text { Externe, Station im Ausland } \\
\text { möglich }\end{array}$ \\
\hline Kassel & $\begin{array}{l}\text { Rotation mit } \\
\text { Fachausbildung }\end{array}$ & 18 & $\begin{array}{l}\text { Wirtschafts-, Politik- } \\
\text { und Gesellschaftswis- } \\
\text { senschaftler (auch FH) }\end{array}$ & Seit Frühjahr 2017 \\
\hline Wolfsburg & $\begin{array}{l}\text { Rotation mit } \\
\text { Fachausbildung }\end{array}$ & 24 & $\begin{array}{l}\text { Absolventen von } \\
\text { Hoch- und Fach- } \\
\text { hochschulen }\end{array}$ & $\begin{array}{l}\text { Feste Einsatzstelle von der } \\
\text { aus Hospitationen in anderen } \\
\text { Stellen erfolgen }\end{array}$ \\
\hline
\end{tabular}


Auf Bundesebene existieren keine Traineeprogramme in der unmittelbaren Verwaltung, allerdings organisiert die Deutsche Gesellschaft für Internationale Zusammenarbeit (GIZ) ein Traineeprogramm in der Entwicklungszusammenarbeit. Zudem führen Institutionen im Finanzsektor, etwa die KfW-Bank oder die Deutsche Bundesbank, Traineeprogramme durch. Im Fall der Bundesbank wird ein solches Programm parallel mit einem Referendariat angeboten. Es werden jedoch nur Absolventen des Referendariats verbeamtet.

In den Bundesländern werden einige Traineeprogramme durchgeführt, die bisherige Wirtschafts-, Verwaltungs- oder Regierungsreferendariate ersetzen, etwa in Berlin, Bremen und Hamburg, oder sich an Volljuristen wenden, wie in Hessen oder Schleswig-Holstein. Berlin führte neben dem Traineeprogramm, das für den höheren Dienst qualifiziert, im Jahr 2016 zudem ein Traineeprogramm für den gehobenen Dienst ein, durch das Bachelorabsolventen rekrutiert werden sollen.

Auf kommunaler Ebene existieren selten echte Traineeprogramme. Häufig findet keine Rotation der Einsatzstationen statt, sondern es erfolgt lediglich eine ergänzende Fortbildung zum normalen Direkteinstieg (etwa Bezirk Neukölln von Berlin, Stadt Ulm). Auch werden Traineeprogramme außerhalb des allgemeinen Verwaltungsdiensts angeboten, beispielsweise zur Vorbereitung auf die Arbeit im Jugend- und Sozialbereich (etwa Stadt Bochum, Stadt Essen, Stadt und Kreis Soest). In den Kommunen werden häufig Bachelorabsolventen oder Absolventen von Fachhochschulen rekrutiert, die entweder gar keine Laufbahnbefähigung erwerben oder im gehobenen Dienst eingruppiert werden.

\subsubsection{Gemeinsamkeiten und Unterschiede öffentlicher und privatwirtschaftlicher Traineeprogramme}

Abgesehen von der unklaren Bezifferung der Traineeprogramme im deutschen öffentlichen Dienst liegen keine Analysen zu deren Qualität vor. Mauch (2011, S. 318) nennt zwar personale und organisationale Voraussetzungen, die gute Traineeprogramme in der öffentlichen Verwaltung erfüllen sollten, es bleibt aber unklar, ob diese auf Ergebnissen zu privatwirtschaftlichen Programmen beruhen oder für den öffentlichen Dienst bestätigt wurden.

Es wurde daher in einer Einzelfallstudie erstmals ein deutsches öffentliches Traineeprogramm untersucht (Fischer 2016). Hierzu wurden leitfadengestützte Interviews mit den Organisatoren des Traineeprogramms geführt und zwei Jahrgänge von Trainees und deren jeweilige direkte Führungskräfte mithilfe eines Web-Surveys quantitativ befragt. Es wurde dabei eine Vollerhebung vorgenommen und die Rücklaufquote betrug 75,5\%. Die Ergebnisse der Studie müssen aber im Licht der dennoch geringen Fallzahl $(\mathrm{N}=69)$ gesehen werden.

Im Folgenden werden die Ergebnisse dieser Studie dargelegt. Es sollen dabei vergleichende Bezüge zu Ergebnissen aus Studien zu privatwirtschaftlichen Traineeprogrammen hergestellt werden, soweit entsprechende Daten verfügbar sind. 


\subsubsection{Aufbau und Ablauf der Traineeprogramme}

Traineeprogramme im öffentlichen Sektor sind zum größten Teil Rotationsprogramme. Einige Programme einige umfassen auch Fachausbildungsphasen (etwa Deutsche Bundesbank, Land Berlin oder Stadt Wolfsburg), in denen der Fokus auf einer der Praxisstationen liegt. Die klassische Konzeption der Programme liegt nahe, weil im Verwaltungskontext, gerade in Laufbahnsystemen, Generalisten gebraucht werden, die eine entsprechende Verwendungsbreite aufweisen. Im privaten Sektor herrschen ebenfalls Rotationsprogramme vor, es existieren aber auch einige ressortbegrenzte Programme (Nesemann 2012).

Die Programme mit Fachausbildungsphase machen jedoch nur Sinn, wenn an der entsprechend vertieften Ausbildung nach dem Traineeprogramm auch Bedarf besteht. Weil im öffentlichen Sektor die Traineeprogramme aber bisher nicht mit einer strategischen Personalbedarfsplanung verbunden sind, ist es fraglich, ob solche Schwerpunkte sinnvoll sind. Das gilt auch, weil erst spät im Programm die Endposition der Trainees festgelegt wird. Dieser Vorwurf wird auch Traineeprogrammen in der Privatwirtschaft gemacht (Konegen-Grenier 1999; Wegerich 2015).

Die Rotation erfolgt in den Programmen im öffentlichen Bereich häufig in drei bis fünf Stationen, in denen die Trainees zwischen drei und sechs Monate arbeiten. Praxisstationen im Ausland sind in einigen wenigen öffentlichen Traineeprogrammen möglich (etwa Land Hamburg oder Ingolstadt). In der Privatwirtschaft hingegen umfassen die Programme häufiger Auslandsstationen (Troy 2007). Insgesamt werden auch hier meist fünf Praxisstationen absolviert und die Programme dauern etwa 18 Monate (Nesemann 2012). Im öffentlichen Dienst dauern Traineeprogramme i. d. R. länger, insbesondere wenn eine Verbeamtung angestrebt und daher eine Laufbahnbefähigung erworben werden soll.

Neben der Ausbildung on-the-job in den Praxisstationen umfassen Traineeprogramme in beiden Sektoren Fortbildungsmaßnahmen off-the-job. Im öffentlichen Sektor sind diese an schon bestehende Verwaltungslehrgänge (etwa Stadt Kassel) oder Qualifizierungsreihen, die den Wirtschaftsreferendaren angeboten wurden (etwa Land Berlin), angelehnt und werden häufig durch die entsprechenden Landesakademien durchgeführt. Das Traineeprogramm des Landes Berlin umfasst auch ein einsemestriges Ergänzungsstudium an der Deutschen Hochschule für Verwaltungswissenschaften in Speyer. Inhalte des Off-the-job-Trainings sind in öffentlichen Traineeprogrammen v. a. die (rechtlichen) Grundlagen von Verwaltungshandeln. Meist sind zusätzlich auch individuell wählbare Schwerpunkte vorgesehen. In privatwirtschaftlichen Programmen ist diese Individualisierung in der theoretischen Ausbildung stärker ausgebildet. Hier existieren oft gar keine festen Curricula, sondern die Fortbildungsinhalte können nach dem Baukastenprinzip zusammengesetzt werden (Troy 2007).

\subsubsection{Ziele und Zielerfüllung}

Traineeprogramme sollen sowohl im privaten als auch im öffentlichen Bereich eine Organisation mit qualifizierten Nachwuchskräften versorgen (Thom und Friedli 2008). Wie dargelegt kann dieses übergreifende Ziel unterteilt werden in (a) eine erfolgreiche Ausbildung der Trainees, (b) deren Sozialisation in der Organisation, (c) Informationsergiebigkeit 
über die Trainees als Beschäftigte, (d) eine Imageverbesserung der anbietenden Organisation und (e) die Nachhaltigkeit des Programms. Auf die Erhebung des Informationsziels wurde verzichtet, weil die beiden Befragtengruppen (Trainees und deren Führungskräfte) dieses nicht einschätzen können, ebenso auf die Untersuchung des Nachhaltigkeitsziels, weil hierüber im konkreten Fall noch keine Aussage getroffen werden kann.

Die Ergebnisse zeigen, dass das untersuchte öffentliche Traineeprogramm die angelegten Ziele erfüllt. Die zusätzlich abgefragte Gesamteinschätzung zum Programm fällt ebenfalls in beiden Befragtengruppen positiv aus. Einige Führungskräfte wünschen sich zudem auf die Frage nach Veränderungsvorschlägen eine Ausweitung des Traineeprogramms, was dieses Ergebnis unterstreicht. Im Mittel bewerten die Führungskräfte die Zielerreichung und das Programm insgesamt positiver als die Trainees. Die Abb. 4.3 zeigt die Verteilung der Bewertungen in den beiden Befragtengruppen.

Wie in Abb. 4.3 zu sehen, wird die Sozialisation der Trainees von den Befragten als am deutlichsten erreicht angesehen. Dies ist Voraussetzung für den weiteren Verbleib der Trainees in der jeweiligen Organisation und trägt so zur Nachhaltigkeit des Programms bei.

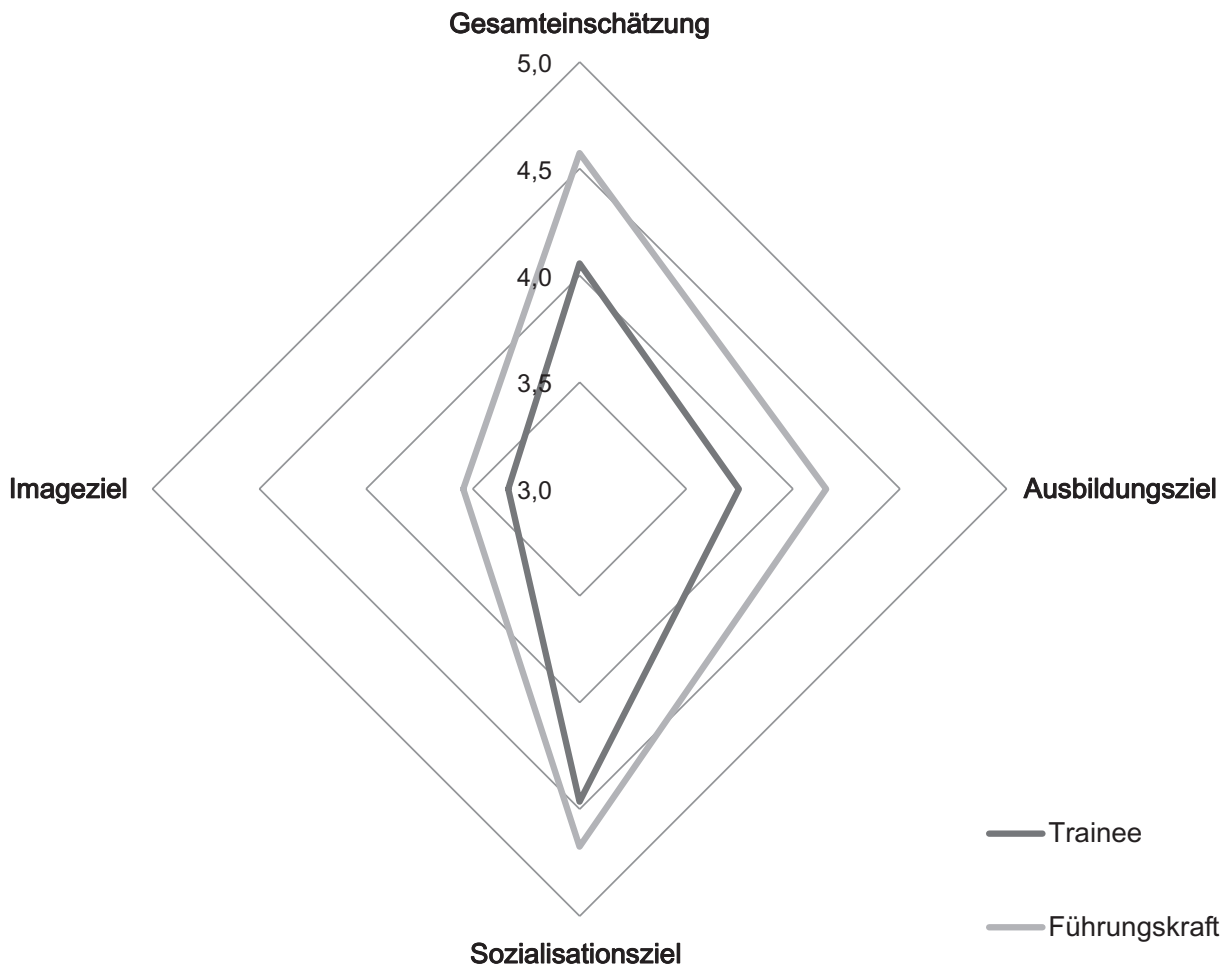

Abb. 4.3 Gesamteinschätzung und Zielerreichung des Traineeprogramms. Abgebildet sind Mittelwerte ( 1 stimme überhaupt nicht zu bis 5 stimme voll und ganz zu); Trainees $(n=28)$, Führungskräfte $(n=41)$ 
Auch die fachliche und persönliche Weiterentwicklung der Trainees wird in beiden Gruppen als hoch bewertet. Die Trainees schätzen das Erreichen des Ausbildungsziels jedoch durchweg schlechter ein, besonders im Bereich der methodischen und sozialen Ausbildung (Abb. 4.4). Diese selbstkritische Einschätzung der Trainees zeigt, dass diese sich insgesamt einen höheren Zugewinn an Kenntnissen und Fertigkeiten erhofft hatten. Im Bereich der fachlichen Ausbildung wird zudem die Verbreiterung des Wissens der Trainees schlechter bewertet als dessen Vertiefung, was der beabsichtigten Entwicklung der Nachwuchskräfte zu Generalisten entgegensteht.

Das Imageziel wird von den Befragten nur knapp positiv eingeschätzt. Das Teilziel der Verbesserung des externen Images des Programmanbieters als Arbeitgeber wird sogar als eher nicht erreicht bewertet (Abb. 4.5). Dass keine sonderliche Außenwirkung besteht, verwundert jedoch nicht, da das Programm in der Öffentlichkeit nicht beworben wird. Das birgt allerdings die Gefahr rückläufiger Bewerberzahlen. Der verwaltungsinterne Ruf des Traineeprogramms wird von den Befragten wiederum als gut eingestuft. Das heißt, andere Verwaltungsmitarbeiter sind, zumindest aus Sicht der Befragten, diesem gegenüber positiv eingestellt. Die Akzeptanz des Programms spricht für die Passfähigkeit des Traineeprogramms zur untersuchten Verwaltung.

Diese Ergebnisse decken sich mit denen von Thom und Ritz (2008), wonach Organisationen, die zu Instrumenten der Personalentwicklung befragt wurden und angaben Traineeprogramme durchzuführen, diese zu $86 \%$ als eher erfolgreich und zu $14 \%$ als sehr erfolgreich einstuften. Saner-Schär (2009) erhob im Rahmen einer Befragung von deutschen Unternehmen, die Traineeprogramme anbieten, wie viele Trainees im Unternehmen verbleiben und welchen Anteil ehemalige Trainees unter den Führungskräften ausmachen. Demnach bleiben in etwas mehr als der Hälfte der befragten Firmen

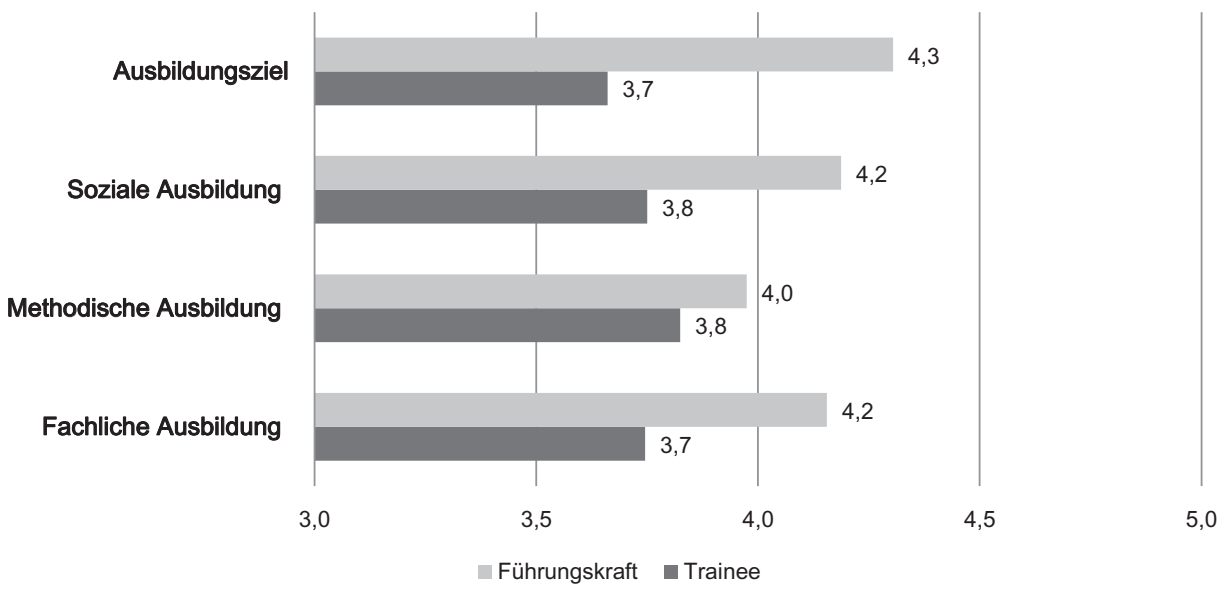

Abb. 4.4 Einschätzung des Ausbildungsziels und Teilziele. Abgebildet sind Mittelwerte (1 stimme überhaupt nicht zu bis 5 stimme voll und ganz zu); Trainees $(n=28)$, Führungskräfte $(n=41)$ 


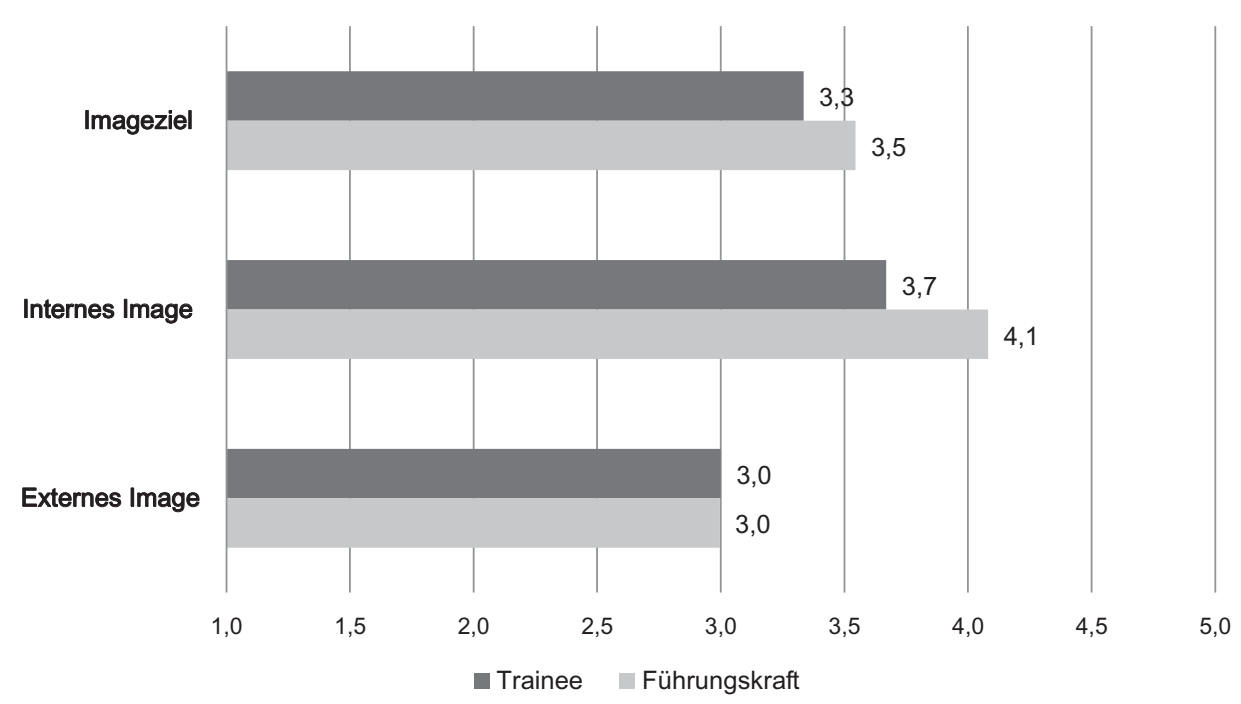

Abb. 4.5 Einschätzung des Imageziels und Teilziele. Abgebildet sind Mittelwerte (1 stimme überhaupt nicht zu bis 5 stimme voll und ganz zu); Trainees $(n=28)$, Führungskräfte $(n=41)$

Trainees auch noch fünf Jahre nach Programmende im Unternehmen. Dass ehemalige Trainees zu Führungskräften aufgestiegen sind, berichten jedoch nur etwa ein Drittel der Unternehmen. Saner-Schär merkt an, dass Unternehmen Traineeprogramme weniger zur Identifikation von Führungspotenzial nutzen, als für ein fachliches Training nach dem Studium. Das Verhältnis von Kosten und Nutzen der Programme ist dann jedoch fraglich.

\subsubsection{Erfolgsfaktoren}

Was gute Traineeprogramme ausmacht, haben für den deutschsprachigen Raum einige empirische Studien zu solchen Programmen in der Privatwirtschaft untersucht und entsprechenden Erfolgsfaktoren aufgezeigt (Meyer-Riedt 1993; Gulden 1996; Schuhen 2011; Nesemann 2012). Traineeprogramme im öffentlichen Dienst sind dagegen bisher kein Teil wissenschaftlicher Analyse. International existieren jedoch einige Fallstudien und Vergleiche von Programmen, wie dem britischen ,fast stream“ (Garrow und Hirsh 2008; Macfarlane et al. 2012).

Die in diesen verschiedenen Studien identifizierten Erfolgsfaktoren von Traineeprogrammen können in drei Faktorengruppen strukturiert werden: personelle, programmspezifische und institutionelle Erfolgsfaktoren. Aus diesen drei Gruppen wurden in der durchgeführten Studie potenzielle Einflüsse ausgewählt, erhoben und die Wirkung auf die gemessene Zielerfüllung des Traineeprogramms analysiert. In der Formulierung der Items für beide Variablengruppen wurde aus Gründen der Vergleichbarkeit zu einem großen Teil auf die Studie von Nesemann (2012) zurückgegriffen, die Traineeprogramme in der Privatwirtschaft in Deutschland, der Schweiz und Österreich untersucht hat. In den Tab. 4.2 und 4.3 sind die Ergebnisse der Regressionsanalysen der Einflussfaktoren auf die Zieldimensionen von Traineeprogrammen verzeichnet. 
Tab. 4.2 Zentrale Einflussfaktoren auf die Gesamteinschätzung und die Einschätzung der Zielerreichung des Programms durch die Führungskräfte (Ordinary-Least-Squares-Regression). Es wurde zusätzlich auf folgende Variablen kontrolliert: Berufserfahrung der Trainees ist wichtig, Verwaltungserfahrung der Trainees ist wichtig, Häufigkeit Kommunikation mit Trainee, Forderung nach mehr Einsatz der Hausleitung, Forderung nach mehr Einsatz der Programmverantwortlichen, Einsatz Programmverantwortliche, Veränderungsbereitschaft, Partizipation bei Programmkonzeption, Jahrgang, Verwaltungsebene, Alter, Geschlecht. $n=36$; ++ Beta-Koeffizienten $>0,5$ mit $p<0,05$; + Beta-Koeffizienten $>0,3$ aber $<0,5$ mit $p<0,05$. (Nach Fischer 2016, S. 258)

\begin{tabular}{l|l|l|l|l}
\hline & Gesamteinschätzung & Ausbildungsziel & Sozialisationsziel & Imageziel \\
\hline $\begin{array}{l}\text { Einsatz Behördenleitung } \\
\begin{array}{l}\text { Fachliche Qualifikation } \\
\text { der Trainees ist wichtig }\end{array}\end{array}$ & ++ & ++ & & ++ \\
\hline $\begin{array}{l}\text { Nichtjuristen sind wich- } \\
\text { tige Nachwuchskräfte }\end{array}$ & + & & & \\
\hline $\begin{array}{l}\text { Ausreichend Zeit zur } \\
\text { Kommunikation mit } \\
\text { Trainee }\end{array}$ & & + & & \\
\hline $\begin{array}{l}\text { Kommunikation zum } \\
\text { Programm }\end{array}$ & & + & & \\
\hline
\end{tabular}

Tab. 4.3 Zentrale Einflussfaktoren auf die Gesamteinschätzung und die Einschätzung der Zielerreichung des Programms durch die Trainees (Ordinary-Least-Squares-Regression). Es wurde zusätzlich auf folgende Variablen kontrolliert: Berufserfahrung, Feedback geben in Praxisstationen, Effektivität Mentoring, Ankunft vorbereitet, Jahrgang, Verwaltungsebene, Geschlecht. $n=27$; ++ Beta-Koeffizienten $>0,5$ mit $p<0,05$; + Beta-Koeffizienten $>0,3$ aber $<0,5$ mit $p<0,1$; Beta-Koeffizienten $<-0,3$ mit $p<0,1$. (Nach Fischer 2016, S. 259)

\begin{tabular}{l|l|l|l|l}
\hline & Gesamteinschätzung & Ausbildungsziel & Sozialisationsziel & Imageziel \\
\hline Projektarbeit & & ++ & + & \\
\hline $\begin{array}{l}\text { Effektivität Off-the-job- } \\
\text { Training }\end{array}$ & & & ++ & \\
\hline $\begin{array}{l}\text { Kommunikation zum } \\
\text { Programm }\end{array}$ & + & & & ++ \\
\hline $\begin{array}{l}\text { Klare Struktur des Pro- } \\
\text { gramms }\end{array}$ & + & & & \\
\hline $\begin{array}{l}\text { Häufigkeit Kommunika- } \\
\text { tion mit Führungskraft }\end{array}$ & & & + & \\
\hline Verwaltungserfahrung & & - & & \\
\hline
\end{tabular}

\section{Personelle Faktoren}

Personengruppen, die Einfluss auf den Erfolg eines Traineeprogramms haben können, sind, neben den Programmverantwortlichen in der Personalabteilung, die Trainees selbst, deren direkte Führungskräfte, Kollegen und die Organisationsleitung. 
In den Ergebnissen sticht der Einfluss des Einsatzes der jeweiligen Behördenleitung besonders heraus. Führungskräfte von Trainees, deren Behördenleitung sich für das Traineeprogramm einsetzt, schätzen den Erfolg des Programms höher ein. Der Einsatz der Leitung wirkt dabei besonders auf die Einschätzung des Imageziels des Traineeprogramms. Das heißt, je stärker sich die Hausleitung einer Behörde für das Programm interessiert und einsetzt, desto besser ist der interne Ruf des Programms und desto besser wird der externe Ruf des Programmanbieters als Arbeitgeber eingeschätzt (Garrow und Hirsh 2008). Dieses Ergebnis bestätigt Annahmen zur symbolischen Führung, die davon ausgehen, dass die Organisationsleitung ,einen wesentlichen Einfluss auf die ganzheitliche Perzeption, welche die Mitarbeitenden vom Programm haben, ausübt" (Nesemann 2012, S. 81). Zudem deckt sich dieses Ergebnis mit denen zu privatwirtschaftlichen Traineeprogrammen. Nesemann (2012) hat hier ebenfalls einen positiven Einfluss des Einsatzes der Unternehmensleitung auf das interne Image eines Programms festgestellt.

Die durchgeführte Befragung hat zudem den Einfluss der Führungskräfte auf den Erfolg des Traineeprogramms aufgezeigt. Es wird in beiden Befragtengruppen ein Effekt des Umfangs der Betreuung des Trainees durch die direkte Führungskraft deutlich. Wenn die Führungskräfte die hierfür zur Verfügung stehende Zeit als ausreichend einstufen, so schätzen sie insbesondere das Ausbildungsziel des Traineeprogramms besser ein. Trainees, die einen hohen Betreuungsumfang durch die Führungskräfte angaben, schätzten v. a. ihre eigene Sozialisation in der jeweiligen Behörde als stärker ein. Es merkten jedoch einige Führungskräfte an, zu wenig Zeit zur Betreuung der Trainees zur Verfügung zu haben. Nesemann (2012) zeigte in ihrer Befragung der Verantwortlichen für privatwirtschaftliche Traineeprogramme ebenfalls auf, dass sich der Einsatz der Führungskräfte auf die Sozialisation der Trainees und deren fachliche Ausbildung auswirkt. Beide Ergebnisse bestätigen ganzheitliche Konzepte von Talent Management. Demnach ist die Durchführung von Traineeprogrammen ein kooperativer Prozess zwischen Personalabteilung, die die Programme plant, und den direkten Führungskräften, die die eigentliche Ausbildung und Entwicklung der Trainees übernehmen (Ritz und Sinelli 2011).

In der Befragung der Führungskräfte des untersuchten öffentlichen Traineeprogramms wurde deutlich, dass diese Wert auf die fachliche Qualifikation der Trainees legen und diese als grundlegend für den Erfolg des Traineeprogramms einstufen. Je wichtiger die Führungskräfte die fachliche Qualifikation der Trainees vor dem Programm einschätzten, desto zufriedener waren sie mit dem untersuchten Traineeprogramm und desto besser schätzten sie den Ausbildungserfolg des Programms ein. Dieser Effekt zeigt eine hohe Erwartungshaltung der Führungskräfte an die Trainees und deckt sich damit mit Erkenntnissen aus privatwirtschaftlichen Programmen (Gulden 1996).

In der Gruppe der Trainees zeigt sich im Zusammenhang mit deren Qualifikation hingegen ein deutlich negativer Zusammenhang mit dem Erfolg des Traineeprogramms. Je mehr Berufserfahrung im öffentlichen Dienst die Trainees haben, desto schwächer fällt der Ausbildungserfolg des Programms aus. Diese starke negative Wirkung auf die fachliche und persönliche Weiterentwicklung der Trainees ist insofern problematisch, als dass 
die Ausbildung der Trainees Kosten verursacht, die im Fall dieser verwaltungserfahrenen Trainees keinen Mehrwert bringen.

Einen Einfluss der Reaktion der Kollegen auf den Trainee, der konkreten Führungsqualität in der Betreuung des Trainees oder des Einsatzes der Programmverantwortlichen in der Personalabteilung wurden nicht nachgewiesen, was aber dem Design dieser Studie geschuldet sein kann.

\section{Programmspezifische Faktoren}

$\mathrm{Zu}$ den programmspezifischen Einflussfaktoren auf den Erfolg von Traineeprogrammen gehören deren Struktur, Ablauf und einzelnen Elemente, wie etwa Fortbildungsmaßnahmen off-the-job-Trainings oder Mentoring.

Unter den programmspezifischen Einflussfaktoren zeigt sich besonders deutlich die Wirkung von Projektarbeit auf die Ausbildung der Trainees. Solche bereichsübergreifenden Projekte befördern dabei v. a. die Ausbildung und die Sozialisation der Trainees. Zum gleichen Ergebnis kommt auch Nesemann (2012) für privatwirtschaftliche Programme.

Zudem nimmt im untersuchten Traineeprogramm das Off-the-job-Training Einfluss auf den Erfolg des Programms. Eine effektive theoretische Ausbildung während des Traineeprogramms, die den Prinzipien des Erwachsenenlernens entspricht, wirkt sich deutlich auf dessen Gelingen aus. Eine detaillierte Analyse der Bewertung des Off-thejob-Trainings zeigt, dass die Trainees motiviert sind, diese Seminare und Workshops zu besuchen und auch deren Lernziele kennen. Sie können jedoch Gelerntes nur z. T. im Arbeitsalltag anwenden und auch nur z. T. mithilfe der Seminare ihre Arbeitsweise verbessern. Die Möglichkeit der kollegialen Beratung, also das Besprechen von Problemen mit anderen Trainees, wird als wenig hilfreich bewertet. Für privatwirtschaftliche Programme liegen keine Ergebnisse zur Wirkung von Off-the-job-Trainings innerhalb von Traineeprogrammen vor.

Im untersuchten Programm wirkt sich zudem die Struktur des Traineeprogramms, also Klarheit von Inhalt, Ablauf und Ansprechpartnern, auf den Erfolg aus. Wenn die Trainees die Struktur des Programms als klar empfinden, dann sind sie mit diesem zufriedener. Eine klare Struktur erscheint dabei v. a. für klassische Rotationsprogramme entscheidend, bei denen Ansprechpartner mit den Einsatzstationen wechseln.

Ein Einfluss von Mentoring auf den Programmerfolg wurde in der durchgeführten Studie nicht nachgewiesen. Dies deckt sich z. T. auch mit den Ergebnissen zu privatwirtschaftlichen Programmen: Nesemann (2012) stellte keinen Einfluss auf die Ausbildung oder Sozialisation der Trainees, sondern lediglich auf das externe Imageziel fest. Mentoringmaßnahmen entfalten demnach zwar eine positive Außenwirkung, dienen aber intern eher nicht zur Zielerreichung.

Für privatwirtschaftliche Programme liegen zusätzlich Ergebnisse zu Effekten eines Auslandsaufenthalts der Trainees während des Programms vor. Wenig überraschend wirken sich diese auf deren interkulturelle Kompetenz und damit die soziale Ausbildung der Trainees aus (Nesemann 2012). 


\section{Organisationale Faktoren}

Organisationale Spezifika, die den Erfolg eines Traineeprogramms beeinflussen können, sind beispielsweise die Organisationsgröße oder Erfahrungen der Organisation mit Traineeprogrammen. Im Fall öffentlicher Programme erscheint zudem eine Offenheit der Mitarbeiter für diese neue Form der Rekrutierung und Entwicklung von Nachwuchskräften relevant. In der durchgeführten Untersuchung nimmt die selbstberichtete Offenheit der Führungskräfte gegenüber anderen Fachrichtungen, die fast alle Führungskräfte in vollem Maß angeben, einen deutlich positiven Einfluss auf die Gesamteinschätzung des Programms durch die Führungskräfte. Im Umkehrschluss bedeutet das aber auch: wer nicht offen gegenüber Nichtjuristen ist, der ist mit dem Traineeprogramm eher nicht zufrieden. Für privatwirtschaftliche Programme erscheint eine solche Offenheit gegenüber Neuem nicht allzu relevant, weil Traineeprogramme hier keinen Bruch mit der bisherigen Personalmanagementpraxis darstellen. Unabhängig davon sind aber bisher keine Ergebnisse verfügbar, ob und welche Art von Organisationskultur hier Einfluss auf Traineeprogramme nimmt.

Eine solche Offenheit gegenüber einer Veränderung kann durch Change-Management-Maßnahmen erzeugt werden. Auch hier zeigt sich ein deutlich positiver Einfluss auf: je nach Befragtengruppe unterschiedliche Zieldimensionen von Traineeprogrammen (Kock und Burke 2008). Dabei wurde untersucht, ob ausreichend zu Zielen, Ablauf und Erfolgen des Traineeprogramms kommuniziert wird und ob Führungskräfte auf die Betreuung der Trainees vorbereitet wurden. Im Fall der Trainees nimmt dies auf das externe Image und deren Gesamteinschätzung des Programms Einfluss. Bei den Führungskräften wurde ein Effekt auf die von ihnen empfundene Erreichung des Ausbildungs- und des Sozialisationsziels festgestellt.

Für privatwirtschaftliche Programme haben sich insbesondere die Organisationsgröße und die Anzahl der Jahre, die das Programm schon existiert, als Einflussfaktoren herausgestellt. Die Größe der Organisation, gemessen an der Anzahl der Beschäftigten, wirkt sich dabei positiv auf die Ausbildung der Trainees aus (Nesemann 2012). Wie lang eine Organisation schon ein Traineeprogramm anbietet, wirkt sich positiv auf das externe Image aus (Nesemann 2012).

\subsection{Fazit: Trainees sind Talente für die Verwaltung!}

Es wird gelegentlich angenommen, dass Talent Management nicht zum öffentlichen Sektor passt (Garrow und Hirsh 2008; Harris und Foster 2010; Poocharoen und Lee 2013; Powell et al. 2013). Das kann mit den dargelegten empirischen Ergebnissen nicht bestätigt werden - ganz im Gegenteil. Das untersuchte öffentliche Traineeprogramm erfüllt die angelegten Ziele gut und es wurden keine Anzeichen für eine Nichtakzeptanz des Programms aufgrund seiner Exklusivität oder dem Transfer von externem Talent aufgezeigt. Das zeigt beispielsweise das interne Image des untersuchten Programms, die hohe Zufriedenheit der befragten Führungskräfte und positive 
Reaktion der Kollegen auf die Trainees. Es besteht also ein Fit zwischen dem TalentManagement-Instrument Traineeprogramm und der untersuchten öffentlichen Organisation. Dies kann wiederum darauf zurückgeführt werden, dass Traineeprogramme einen Kompromiss zwischen den beiden Rekrutierungsparadigmen „grow“ und „buy“ darstellen. Dieses Instrument des Talent Managements ist daher besonders gut für den öffentlichen Bereich geeignet.

Traineeprogramme sind zudem sinnvoll für den öffentlichen Sektor, weil mit ihnen Generalisten entwickelt werden und die zumeist lange Tätigkeitszeit, die organisationseigene Entwicklung von Talent lohnenswert machen. Dies gilt vor allem, wenn die Trainees später verbeamtet werden. Als problematisch hat sich hingegen die Nutzung von Traineeprogrammen zur internen Personalentwicklung erwiesen. Wurden Personen mit Verwaltungserfahrung als Trainees rekrutiert, wurde ein geringerer Ausbildungserfolg nachgewiesen, sodass sich die Investition insbesondere in kostenintensives Off-the-jobTraining nicht lohnt. Hier müssen andere Wege der Talententwicklung geschaffen werden. Das Traineeprogramm von Ingolstadt zeigt eine mögliche Lösung auf, indem zwei getrennte Programmvarianten für interne und externe Bewerber angeboten werden.

Der Vergleich zwischen privatwirtschaftlichen und öffentlichen Programmen hat deutlich gemacht, dass diese in beiden Sektoren durch ähnliche Faktoren beeinflusst werden. Der Einfluss der Organisationsleitung, der Führungskräfte und von Projektarbeit ist etwa in privatwirtschaftlichen wie in öffentlichen Programmen deutlich. Für den öffentlichen Dienst wurde zudem ein Einfluss der Qualifikation der Trainees, des Off-the-job-Trainings, der Offenheit der Führungskräfte gegenüber Neuem sowie der Kommunikation zum Programm und Vorbereitung der Programmeinführung festgestellt.

Die ähnlichen Einflussfaktoren lassen auf eine Übertragbarkeit von Ergebnissen privatwirtschaftlicher Studien zu Traineeprogrammen auf öffentliche Programme schlieBen. Es scheint, dass eher das konkrete Instrument von Talent Management, nämlich das Traineeprogramm an sich, die Erfolgsfaktoren bestimmt und nicht so sehr das organisationale Setting. Das würde den Annahmen widersprechen, wonach der organisationale Kontext einen erheblichen Einfluss auf die konkrete Ausgestaltung des Talent Managements hat (Vaiman und Collings 2013; Thunnissen et al. 2013b). Dabei muss bedacht werden, dass hier kein Talent-Management-Konzept im Ganzen, sondern lediglich ein einzelnes Instrument untersucht wurde.

Umso wichtiger ist es, dass die hier dargestellten Ergebnisse durch weitere Untersuchungen öffentlicher wie privater Traineeprogramme abgesichert werden. Im weiteren Verlauf ist dabei auch die Analyse der Nachhaltigkeit und Kosteneffizienz der Programme von besonderem Interesse. Unabhängig vom konkreten Instrument Traineeprogramm muss aber auch das Talent Management öffentlicher Stellen im Gesamten empirisch untersucht werden, um tatsächlich darlegen zu können, wie sich hier Wirkmechanismen zwischen öffentlichem und privatem Sektor unterscheiden. Die so identifizierten Faktoren können dann als Stellschrauben wirken, um auf eine bessere Art und Weise ,good people“ für ,good government“ zu finden und zu fördern. 


\section{Literatur}

Ashton, C., \& Morton, L. (2005). Managing talent for competitive advantage. Taking a systemic approach to talent management. Strategic HR Review, 4(5), 28-31. https://doi. org/10.1108/14754390580000819.

Bell, S. T., Villado, A. J., Lukasik, M. A., Belau, L., \& Briggs, A. L. (2011). Getting specific about demographic diversity variable and team performance relationships: A meta-analysis. Journal of Management, 37(3), 709-743. https://doi.org/10.1177/0149206310365001.

Berman, E., Bowman, J., West, J., \& Wart, M. van. (2013). Human resource management in public service. Paradoxes, processes, and problems. Los Angeles: Sage.

Bidwell, M., \& Keller, J. (2014). Within or without? How firms combine internal and external labor markets to fill jobs. Academy of Management Journal, 57(4), 1035-1055. https://doi. org/10.5465/amj.2012.0119.

Björkman, I., Ehrnrooth, M., Mäkelä, K., Smale, A., \& Sumelius, J. (2013). Talent or not? Employee reactions to talent identification. Human Resource Management, 52(2), 195-214. https:// doi.org/10.1002/hrm.21525.

Bogumil, J., \& Jann, W. (2009). Verwaltung und Verwaltungswissenschaft in Deutschland. Einführung in die Verwaltungswissenschaft. Wiesbaden: VS Verlag.

Cappelli, P. (2008). Talent management for the twenty-first century. Harvard Business Review, 86(3), 74-81.

Cappelli, P., \& Keller, J. (2014). Talent management. Conceptual approaches and practical challenges. Annual Review of Organizational Psychology and Organizational Behavior, 1(1), 305-331. https://doi.org/10.1146/annurev-orgpsych-031413-091314.

Collings, D. G., \& Mellahi, K. (2009). Strategic talent management. A review and research agenda. Human Resource Management Review, 19(4), 304-313. https://doi.org/10.1016/j. hrmr.2009.04.001.

Collings, D. G., Scullion, H., \& Vaiman, V. (2015). Talent management. Progress and prospects. Human Resource Management Review, 25(3), 233-235. https://doi.org/10.1016/j. hrmr.2015.04.005.

Cordes, S. O. (2000). Einfluß von Lean-Management und Business Reeingineering auf die Gestaltung von Trainee-Programmen in der Automobilindustrie. Konzeptionelle Grundlagen, Fallstudie und Gestaltungsempfehlungen. Köln: Botermann \& Botermann.

dbb Berlin. (2015a). Besoldungstabelle 2015. Für Beamtinnen und Beamte des Landes Berlin. Hg. v. Landesleitung des dbb - Beamtenbund und Tarifunion - Berlin. Berlin. http://www.dbb.de/ fileadmin/pdfs/einkommenstabellen/besoldungstab_berlin_150801.pdf.

dbb Berlin. (2015b). Entgelttabelle Berlin 2015. Hg. v. Landesleitung des dbb - Beamtenbund und Tarifunion - Berlin. Berlin. http://www.dbb.de/fileadmin/pdfs/einkommenstabellen/150328_ entgelttabelle_tvl_berlin_vorlaeufig.pdf.

Demmke, C. (2011). Die Reform der öffentlichen Dienste im internationalen Vergleich. In R. Koch, P. Conrad, \& H. Lorig (Hrsg.), New Public Service. Öffentlicher Dienst als Motor der Staats- und Verwaltungsmodernisierung (S. 55-79). Wiesbaden: Gabler Verlag.

Dries, N. (2013). The psychology of talent management. A review and research agenda. Human Resource Management Review, 23(4), 272-285. https://doi.org/10.1016/j.hrmr.2013.05.001.

Fischer, C. (2016). Traineeprogramme als innovative Form der Nachwuchsausbildung im öffentlichen Dienst. Verwaltung und Management, 22(5), 250-262. https://doi.org/10.5771/0947-98562016-5-250.

Gallardo-Gallardo, E., Nijs, S., Dries, N., \& Gallo, P. (2015). Towards an understanding of talent management as a phenomenon-driven field using bibliometric and content analysis. Human Resource Management Review, 25(3), 264-279. https://doi.org/10.1016/j.hrmr.2015.04.003. 
Garrow, V., \& Hirsh, W. (2008). Talent management. Issues of focus and fit. Public Personnel Management, 37(4), 389-402. https://doi.org/10.1177/009102600803700402.

Gelens, J., Hofmans, J., Dries, N., \& Pepermans, R. (2014). Talent management and organisational justice. Employee reactions to high potential identification. Human Resource Management Journal, 24(2), 159-175. https://doi.org/10.1111/1748-8583.12029.

Gholipour, Z., Ali, S. S., \& Taghi, M. M. (2015). Investigating the relationship between talent management strategy and career path of principals of schools in Shahr-e-Kord. International Research in Education, 4(1), 39. https://doi.org/10.5296/ire.v4i1.9007.

Glenn, T. (2012). The state of talent management in Canada's public sector. Canadian Public Administration, 55(1), 25-51. https://doi.org/10.1111/j.1754-7121.2012.00204.x.

Görtler, B., \& Gourmelon, A. (2015). Auswahl und Entwicklung von Führungsnachwuchskräften in Kommunalverwaltungen. Verwaltung \& Management, 21(2), 73-82. https://doi. org/10.5771/0947-9856-2015-2-73.

Groysberg, B., Lee, L.-E., \& Nanda, A. (2008). Can they take it with them? The portability of star knowledge workers' performance. Management Science, 54(7), 1213-1230. https://doi. org/10.1287/mnsc.1070.0809.

Gulden, H. (1996). Evaluation von Traineeprogrammen als Alternative zur klassischen Form des Berufseinstiegs. Betrachtung aus Firmen- und Studentensicht. München: Hampp.

Harris, L., \& Foster, C. (2010). Aligning talent management with approaches to equality and diversity. Equality, Diversity and Inclusion: An International Journal, 29(5), 422-435. https://doi. org/10.1108/02610151011052753.

Heller, R., \& Ruf, M. (2009). Talent Management als Instrument der Führungsnachwuchskräfteentwicklung. In W. Jäger \& A. Lukasczyk (Hrsg.), Talent Management. Strategien, Umsetzung, Perspektiven (S. 121-128). Köln: Luchterhand.

Horwitz, S. K., \& Horwitz, I. B. (2007). The effects of team diversity on team outcomes: A metaanalytic review of team demography. Journal of Management, 33(6), 987-1015. https://doi. org/10.1177/0149206307308587.

Jäger, W. (2009). Talent Management ist Personalmanagement. In W. Jäger \& A. Lukasczyk (Hrsg.), Talent Management. Strategien, Umsetzung, Perspektiven (S. 15-24). Köln: Luchterhand.

Kock, R., \& Burke, M. (2008). Managing talent in the South African public service. Public Personnel Management, 37(4), 457-470. https://doi.org/10.1177/009102600803700406.

Konegen-Grenier, C. (Hrsg.). (1999). Traineeprogramme. Berufsstart für Hochschulabsolventen. Institut der Deutschen Wirtschaft. Köln: div Dt. Inst.-Verlag.

Kuhlmann, S., \& Wollmann, H. (2013). Verwaltung und Verwaltungsreformen in Europa. Einführung in die vergleichende Verwaltungswissenschaft. Wiesbaden: Springer VS.

Laegreid, P., \& Wise, L. R. (2007). Reforming human resource management in civil service systems: Recruitment, mobility, and representativeness. In J. C. N. Raadschelders, T. A. J. Toonen, \& F. M. van der Meer (Hrsg.), The civil service in the 21st century. Comparative perspectives (S. 185-200). Basingstoke: Palgrave Macmillan.

Lavigna, R. J., \& Hays, S. W. (2004). Recruitment and selection of public workers. An international compendium of modern trends and practices. Public Personnel Management, 33(3), 237253. https://doi.org/10.1177/009102600403300301.

Lewis, R. E., \& Heckman, R. J. (2006). Talent management. A critical review. Human Resource Management Review, 16(2), 139-154. https://doi.org/10.1016/j.hrmr.2006.03.001.

Lück, T. (2011). Im Bund mit guten Leuten. Personalentwicklung in Bundesbehörden - Ein bestpractice-Vergleich mit den Ländern. Marburg: Tectum.

Macfarlane, F., Duberley, J., Fewtrell, C., \& Powell, M. (2012). Talent management for NHS managers. Human resources or resourceful humans? Public Money \& Management, 32(6), 445-452. https://doi.org/10.1080/09540962.2012.728786. 
Mauch, S. (2011). Rekrutierung und Personalauswahl. In B. Blanke, F. Nullmeier, C. Reichard, \& G. Wewer (Hrsg.), Handbuch zur Verwaltungsreform (S. 311-320). Wiesbaden: VS Verlag.

Meyer-Riedt, T. (1993). Trainee-Programme für Nachwuchskräfte mit Hochschulabschluß und Sonderausbildungsprogramme für Abiturienten. Zur Praxis der Nachwuchskräfteentwicklung in qualifizierten betriebswirtschaftlichen Tätigkeiten. Köln: Botermann \& Botermann.

Meyers, M. C., Woerkom, M. van, \& Dries, N. (2013). Talent - Innate or acquired? Theoretical considerations and their implications for talent management. Human Resource Management Review, 23(4), 305-321. https://doi.org/10.1016/j.hrmr.2013.05.003.

Nerdinger, F. W. (2014). Gravitation und organisationale Sozialisation. In F. W. Nerdinger, G. Blickle, \& N. Schaper (Hrsg.), Arbeits- und Organisationspsychologie (S. 71-82). Berlin: Springer.

Nesemann, K. (2012). Talentmanagement durch Trainee-Programme. Auswirkungen der Gestaltungsmerkmale auf den Programmerfolg. Wiesbaden: Gabler.

Poocharoen, O., \& Lee, C. (2013). Talent management in the public sector. A comparative study of Singapore, Malaysia, and Thailand. Public Management Review, 15(8), 1185-1207. https://doi. org/10.1080/14719037.2013.816525.

Powell, M., Duberley, J., Exworthy, M., Macfarlane, F., \& Moss, P. (2013). Has the British National Health Service (NHS) got talent? A process evaluation of the NHS talent management strategy? Policy Studies, 34(3), 291-309. https://doi.org/10.1080/01442872.2013.798533.

Reichard, C. (2013). Qualifikation der Ressource Personal. In C. Reichard \& E. Schröter (Hrsg.), Zur Organisation öffentlicher Aufgaben. Effizienz, Effektivität und Legitimität (S. 286-302). Opladen: Budrich.

Reichard, C., \& Röber, M. (2012). Ausbildung der Staatsdiener von morgen. Bestandsaufnahme Reformtendenzen - Perspektiven. Berlin: Ed. Sigma (40).

Reichard, C., \& Schröter, E. (2009). Der öffentliche Dienst im Wandel der Zeit: Tradierte Probleme, aktuelle Herausforderungen und künftige Reformperspektiven. der moderne staat - Zeitschrift für Public Policy, Recht und Management, 2(1), 17-36. https://doi.org/10.3224/dms. v2i1.2866.

Rippler, S., Woischwill, B., \& Klönne, L. (2013). Recht einfach: Rahmenbedingungen, Verträge und Co. In B. Woischwill, L. Klönne, \& S. Rippler (Hrsg.), Trainee-Knigge (S. 7-18). Wiesbaden: Springer Fachmedien.

Ritz, A., \& Sinelli, P. (2011). Talent Management - Überblick und konzeptionelle Grundlagen. In A. Ritz \& N. Thom (Hrsg.), Talent Management. Talente identifizieren, Kompetenzen entwickeln, Leistungsträger erhalten (S. 3-23). Wiesbaden: Springer Fachmedien.

Ritz, A., \& Thom, N. (2011). Talent Management auf dem Prüfstand - was Sie für Ihren Führungsalltag wissen müssen. In A. Ritz \& N. Thom (Hrsg.), Talent Management. Talente identifizieren, Kompetenzen entwickeln, Leistungsträger erhalten (S. 235-252). Wiesbaden: Springer Fachmedien.

Rutledge, L., LeMire, S., Hawks, M., \& Mowdood, A. (2016). Competency-based talent management: Three perspectives in an academic library. Journal of Library Administration, 56(3), 235-250. https://doi.org/10.1080/01930826.2015.1105051.

Saner-Schär, M. C. (2009). Trainee-Programme für Absolventen der Wirtschaftswissenschaften in Deutschland. Konzeptionelle Grundlagen, empirische Erhebung, state of the art (2. Aufl.). Bern: IOP (Arbeitsbericht/Institut für Organisation und Personal der Universität Bern).

Scholz, C. (2000). Personalmanagement. Informationsorientierte und verhaltenstheoretische Grundlagen. München: Vahlen.

Schuhen, M. (2011). Führungsnachwuchs mit System. Planung und Gestaltung einer Lernumgebung für Trainee-Programme. Marburg: Tectum.

Sheehan, M., \& Anderson, V. (2015). Talent management and organizational diversity. A call for research. Human Resource Development Quarterly, 26(4), 349-358. https://doi.org/10.1002/ hrdq. 21247. 
Smith, R. (2015). Talent management. Building the case for direct entry into leadership roles in British policing. The Police Journal, 88(2), 160-173. https://doi.org/10.1177/0032258X15579357.

Suk Kim, P., \& Kotchegura, A. (2016). Talent management in government in times of economic instability. Selected cases from the BRICS countries. Public Money \& Management, 37(1), 7-14. https://doi.org/10.1080/09540962.2016.1249223.

Thom, N. (1987). Personalentwicklung als Instrument der Unternehmungsführung. Konzeptionelle Grundlagen und empirische Studien. Stuttgart: Poeschel.

Thom, N., \& Friedli, V. (2008). Hochschulabsolventen gewinnen, fördern und erhalten. Bern: Haupt.

Thom, N., \& Ritz, A. (2008). Public management. Wiesbaden: Springer Fachmedien.

Thunnissen, M., Boselie, P., \& Fruytier, B. (2013a). A review of talent management. 'infancy or adolescence?'. The International Journal of Human Resource Management, 24(9), 1744-1761. https://doi.org/10.1080/09585192.2013.777543.

Thunnissen, M., Boselie, P., \& Fruytier, B. (2013b). Talent management and the relevance of context. Towards a pluralistic approach. Human Resource Management Review, 23(4), 326-336. https://doi.org/10.1016/j.hrmr.2013.05.004.

Troy, I. (2007). Trainee-Programme für Hochschulabsolventen. Eine Strukturanalyse zur Programmplanung mit einer empirischen Untersuchung zur Identifizierung von Programmtypen. Diplomarbeit, Universität Augsburg.

Vaiman, V., \& Collings, D. G. (2013). Talent management. Advancing the field. The International Journal of Human Resource Management, 24(9), 1737-1743. https://doi.org/10.1080/0958519 2.2013.777544.

Wegerich, C. (2015). Strategische Personalentwicklung in der Praxis. Berlin: Springer.

\section{Über die Autorin}

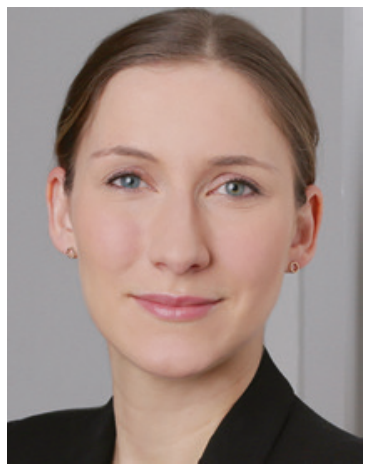

Caroline Fischer ist wissenschaftliche Mitarbeiterin und Doktorandin am Lehrstuhl für Public und Non-Profit Management an der Universität Potsdam. Dort forscht sie u. a. zum Personalmanagement im öffentlichen Sektor. Sie studierte Politik- und Kommunikationswissenschaft an den Universitäten Dresden und Wrocław und schloss an der Universität Potsdam mit einem Master of Arts in Verwaltungswissenschaft ihr Studium ab. 